\title{
CARACTERÍSTICAS DEL ADULTO MAYOR QUE ACEPTA SER VACUNADO CONTRA LA INFLUENZA EN SAN LUIS TLAXIALTEMALCO - 2004.
}

Mtro. Virginio Reyes Audiffred, Lic Inés Gabriela Escobar Moreno*, Lic. Ima Castillo Sánchez**, Lic. Ma. Eva Flores Sänchez, Lic. Angélica Alcóntara Méndez** Lic Ima Lozano Montes De Oca*, Roxana Montaño Humphrey**. Mo Guadolupe Escobedo Acosta**:

" Coordinadora de la Especialidad de Enfermeria del Anciano DEP. ENEO-UNAM, **Egresadas de la Especialidad de Enfermeria del Anciano 2005

\section{RESUMEN}

La influenza es una enfermedad aguda viral en las vías respiratorias altas capaz de provocar complicaciones potencialmente letales en pacientes ancianos. La vacunación anual es la intervención de salud más importante para reducir el impacto de la influenza. Existen diversos factores que influyen para la aceptación o rechazo de la aplicación de la vacuna. El propósito de este estudio fué determinar las características de la población que acepta la vacuna de la influenza e identificar grupos de población que presentan riesgo, especialmente de no vacunarse en la comunidad de San Luis Tlaxialtemalco. El estudio fué descriptivo y transversal. Con una muestra no probabilistica y conformada por 48 personas de 60 y más años de edad. Se aplicó una encuesta estructurada de 25 preguntas y diferentes escalas. En cuanto a los resultados, el $84.48 \%$ aceptó la aplicación de la vacuna de la influenza. El 81.3\% desconoce la enfermedad de la influenza, el $77.1 \%$ cree que la influenza es grave, el $64.6 \%$ desconocía la vacuna contra la influenza, el $97.9 \%$ cree que previene la influenza y el $85.4 \%$ cree que la vacuna no le ocasionará problemas. Los factores que influyeron para la aceptación de la vacuna y en cuanto a quien se la recomendó el $37.3 \%$ refieren que fué la Enfermera y al $43.8 \%$ nadie.

Palabras clave: vacuna contra la influenza; aceptación ó rechazo de la vacuna contra la influenza; adulto mayor en riesgo.

\section{ABSTRACT.}

Influenza is a high respiratory tract viral accute disease able to bring about potentially fatal complications in elderly patients. The annual vaccination is the most important health intervention for decreasing the flu impact. There are several factors influencing the vaccine application acceptance or rejection. This study aims determining characteristics of the population that accepts the influenzo voccine and identifying in-risk population groups in the event they don't receive it within San Luis Tlaxialtemalco area. It was a descriptive transversal study with a no-probabilistic sample including 48 people, 60 or more years old. A structured survey with 25 questions and different scales. Regarding outcomes, $84.48 \%$ accepted the influenzo vaccine application. The $81.3 \%$ are not familiar with influenza disease, $77.1 \%$ believes the flu is grave, $64.6 \%$ is not familiar with the influenza vaccine, $97.9 \%$ believes they are taking preventive octions for the influenza, and $85.4 \%$ thinks the influenzo voccine will not cause them any problems, which is influencing on the vaccine acceptance. Regarding the person who recommended them the influenza vaccine, $31.3 \%$ said was the nurse and $43.8 \%$ said nobody did.

Key words: Influenza vaccine; Influenza vaccine acceptance or rejection; Elderly adult population in risk. 


\section{INTRODUCCIÓN}

La influenza es una enfermedad aguda viral de las vías respiratorias, que se caracteriza por fiebre, cefalalgia, mialgia, postración, coriza, dolor de garganta y tos. Es altamente contagiosa a través de pequeñas gotas de secreciones respiratorias por lo que se convierte rápidamente en una epidemia. En grupos vulnerables como niños y ancianos la morbilidad es elevada y capaz de provocar complicaciones potencialmente letales.

En México la influenza se reporta como la novena causa de muerte en la población general, en 1999 ocasionó 17 519 defunciones, 44.5\% de éstas se registraron en la población mayor de 65 años de edad, grupo en que ocupó el séptimo lugar.'

La vacunación anual para personas de alto riesgo es la acción más importante para reducir el impacto de la influenza. Sin embargo, la cobertura de vacunación es variable de un pais a otro. En Estados Unidos, el 58\% de personas de 65 y más años declaró haber sido vacunado en los últimos 12 meses, $^{2}$ en Italia el nivel de vacunación se ha descrito como inapropiado siendo del $26-49 \%,{ }^{3}$ en Inglaterra en personas residentes de la comunidad el rango de vacunación fue de $44-59 \% \%^{4}$ y en España la cobertura calculada en personas de 65 y más años vario de 1993 a 1999 año con año del 35\% $0^{5}$ al 94\%. ${ }^{6}$

Los factores que influyen para aceptar o rechazar la aplicación de la vacuna de la influenza son muy diversos. En España, se analizó un conjunto de variables demográficas en adultos mayores, tales como el nivel socioeconómico, estilos de vida, estado de salud, y utilización de los senvicios de salud. Se concluyó que no existe asociación entre el nivel socioeconómico y la vacunación de la influenza en personas mayores de 65 años. 7 En Suiza, un estudio reportó que las razones para rehusar la vacuna contra la influenza en los ancianos fueron: la creencia subjetiva del paciente sobre su estado de salud y susceptibilidad hacia la influenza, así como la opinión acerca de la eficacia de la vacuna. 8 Otra investigación en Canadá informó que entre los factores predictivos para la vacunación de la influenza, se encuentran: ser casado, contar con educación superior, tabaquismo y alcoholismo positivos, percepción del estado de salud como bueno, ejercicio regular, vida urbana, estado cognitivo conservado y comorbilidad. ${ }^{9}$

En México no se encontró información sobre la cobertura de la vacuna de la influenza, tampoco de los factores que influyen para su aplicación en la población de adultos mayores. Este estudio se desarolla con el propósito de determinar las características de la población que acepta la vacuna de la influenza e identificar grupos de población que presentan riesgo, especialmente de no vacunarse en la comunidad de San Luis Tlaxialtemalco.

\section{MÉTODOS}

Se realizó un estudio descriptivo y transversal, de Septiembre a Diciembre del 2004. Como estrategias para la aplicación de la vacuna se utilizo la colocación de un puesto de vacunación y la visita domiciliaria.

Se entrevistó en total a cincuenta y ocho personas de sesenta y más años de edad, de las tres áreas geoestadísticas básicas (AGEBS), del pueblo de San Luis Tlaxiatemalco. Los criterios de inclusión: edad de 60 años ó más, vivir en San Luis Talxialtemalco, aceptación voluntaria para contestar la encuesta y la aplicación de la vacuna. Criterios de exclusión: Edad menor a los 60 años cumplidos, presentar deterioro intelectual severo, no ser residente de San Luis Tlaxialtemalco y no aceptar voluntariamente contestar la encuesta y la aplicación de la vacuna. Criterios de eliminación: Contestación incompleta de la encuesta. Se excluyó a diez adultos mayores que no aceptaron la aplicación de la vacuna y/o contestar la encuesta. La muestra fué no probabilística y por conveniencia e incluyó a cuarenta y ocho adultos mayores.

La información fué obtenida a través de un instrumento de veinticinco ítems, y cuatro escalas de evaluación geriátrica: Evaluación del Estado Mental, Actividades Básicas de la Vida Diaria (ABVD), Actividades Instrumentales de la Vida Diaria (AIVD) y Depresión Geriátrica.

A través de la encuesta se midieron variables como: características demográficas, capacidad funcional, riesgos a la salud y enfermedades, la utilización de los senvicios de salud así como la información que tenía este grupo de población acerca de la influenza y la vacuna. Se cuidó el anonimato de la población de estudio, y la aceptación de su participación voluntaria. Los datos fueron procesados en el programa SPSS versión 11.0 para su análisis.

\section{RESULTADOS}

De 58 adultos mayores a los que se ofreció la vacuna, 49 (84.48\%) aceptaron la aplicación, uno de estos se eliminó de la investigación por no contestar la encuesta. En total se incluyeron $N=48$, el $75 \%$ pertenecen al grupo de edad de 60 a 74 años, el $75 \%$ son mujeres, el $45.8 \%$ no tiene estudios, el $47.9 \%$ cursó de 1 a 6 años de escolaridad y solo el $6.3 \%$ tiene de 7 a 9 años de estudio. En cuanto al estado civil, el $54.2 \%$ son casados y el $41.7 \%$ son viudos. (Tabla I) 
Tabla I

CARACTERISTICAS SOCIO-DEMOGRAFICAS DE LOS ADULTOS MAYORES DE SAN LUIS TLAXIALTEMALCO, DF., QUE ACEPTARON LA APLICACIÓN DE LA VACUNA CONTRA LA INFLUENZA. JUNIO 2005.

\begin{tabular}{|lrrr|}
\hline VARIABLE & FEMENINO & MASCULINO & TOTAL \\
& $\mathrm{N}=36$ & $\mathrm{~N}=12$ & $\mathrm{~N}=48$ \\
GENERO & $\%$ & $\%$ & 100.0 \\
GRUPO DE EDAD & 75.0 & 25.0 & \\
60 a 74 años & & & 75.0 \\
75 y más años & 60.4 & 14.6 & 25.0 \\
ESTADO CIVIL & 14.6 & 10.4 & 2.1 \\
Soltero & & & 54.2 \\
Casado & 2.1 & 0.0 & 41.7 \\
Viudo & 35.4 & 18.8 & 2.1 \\
No respondió & 35.4 & 6.3 & 0.0 \\
\hline
\end{tabular}

FUENTE: Encuestas aplicadas a los adultos mayores de San Luis Tlaxialtemalco, D.F, Septiembre 2004

El $93.8 \%$ presentan su función mental sin deterioro, el $60.4 \%$ no presenta depresión y el $83.4 \%$ percibe su salud de buena a excelente.

En relación a las enfermedades crónico degenerativas encontramos que el $37.5 \%$ presenta hipertensión arterial, el $10.4 \%$ presenta artritis y el $22.9 \%$ presenta Diabetes Mellitus. Con respecto a la ingesta del número de medicamentos el $39.6 \%$ no ingiere, y el $60.4 \%$ consume algún medicamento. El 91.7\% no presenta el hábito de fumar.

En cuanto a las Actividades Básicas de la Vida Diaria (ABVD), el 79.2\% es independiente y el 64.4\% es independiente en las Actividades Instrumentales de la vida Diaria (AIVD).

En relación al servicio médico el 29.2\% pertenece al ISSSTE, el $22.9 \%$ al IMSS, el $16.7 \%$ cuentan con servicio médico particular, y el 31.3\% no cuentan con ninguno.

Respecto a la información que tenían acerca de la influenza: el $81.3 \%$ no sabía de la enfermedad y el $77.1 \%$ cree que la influenza es grave. En lo que se refiere a la última fecha en que se aplicaron la vacuna, el 12.5\% lo hizo hace un año, $4.2 \%$ hace 2 años y el $83.3 \%$ reportaron no haber recibido una vacunación contra la influenza. Con respecto al conocimiento de la vacuna, el $64.6 \%$ no la conocía (Fig. 1), el 97.9\% cree que previene la influenza (Fig. 2) y el $85.4 \%$ cree que la vacuna no les ocasionará problemas.

En cuanto a quien le recomendó la misma, el 2.1\% refiere que fué el médico, el $31.3 \%$ la Enfermera y el $43.8 \%$ dice que nadie. (Fig.3)

Fig.1

CONOCIMIENTO DE LA VACUNA CONTRA LA INFLUENZA EL ADULTO MAYOR DE SAN LUIS TLAXIAITEMALCO, DF., JUNIO 2005

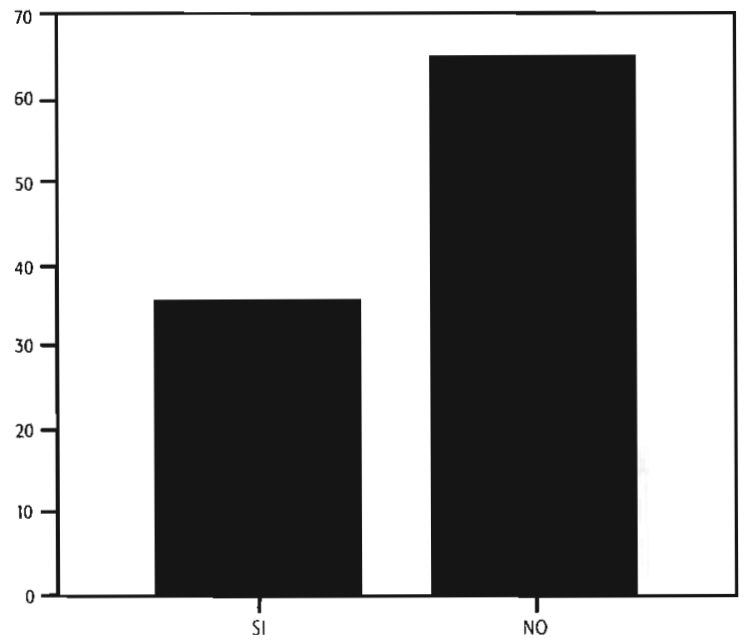

FUENTE: Encuestas aplicadas a los adultos mayores de San Luis Tlaxialtemalco, D.F, Septiembre 2004 
Fig.2

\section{CREENCIA DE QUE QUE LA VACUNA PREVIENE LA \\ INFLUENZA AL ADULTO MAYOR DE SAN LUIS \\ TLAXIALTEMALCO, DF., JUNIO 2005}

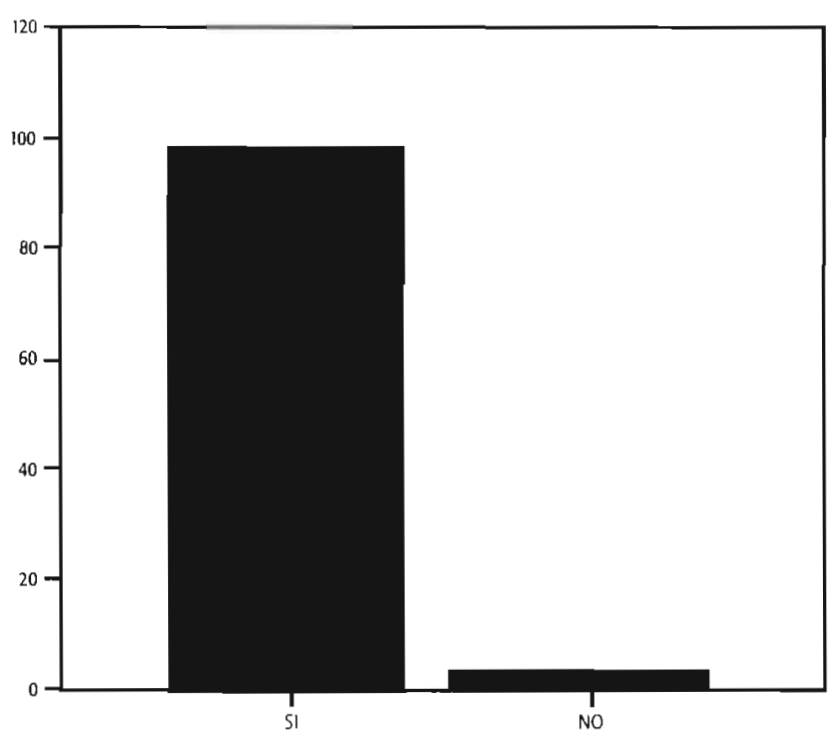

FUENTE: Encuestas aplicadas a los adultos mayores de San Luis Tlaxialtemalco, DF, Septiembre 2004

Fig.3

RECOMENDACIONES DE LA APLICACIÓN DE VACUNA CONTRA LA INFLUENZA AL ADULTO MAYOR DE SAN LUIS TLAXIALTEMALCO, DF., JUNIO 2005

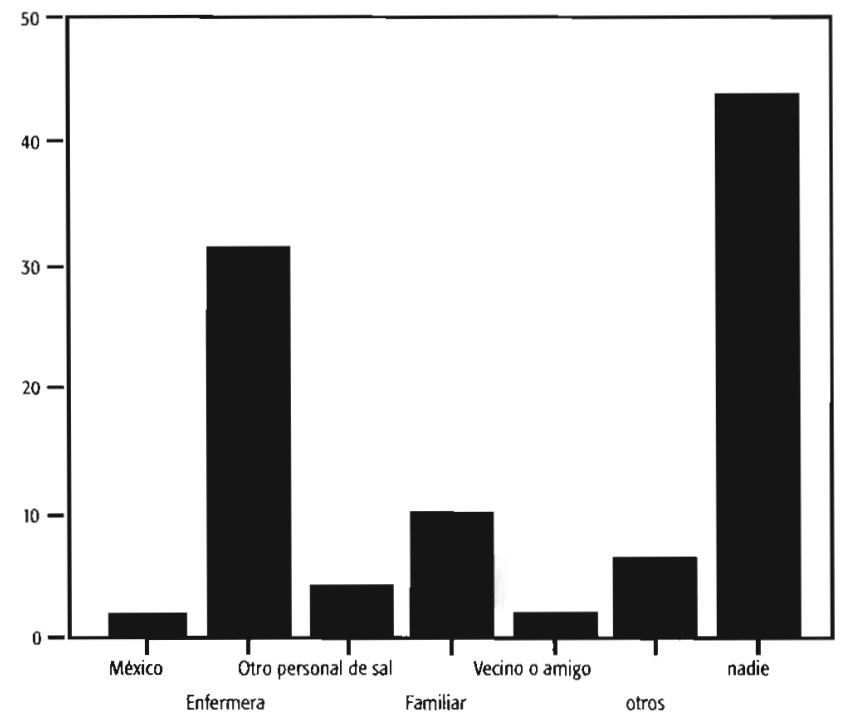

FUENTE: Encuestas aplicadas a los adultos mayores de San Luis Tlaxialtemalco, D.F, Septiembre 2004.

\section{DISCUSIÓN}

El porcentaje de rechazo a la vacunación contra la influenza fué bajo, el 15.5\%. Investigaciones citadas por Lawrence (2004) reportan que la tasa de prevalencia para el rechazo a recibir la vacunación varía entre el 9 y $66 \%{ }^{8}$

Entre los factores demográficos asociados al adulto mayor que aceptó la aplicación de la vacuna se encontraron: el mayor porcentaje son mujeres en edades entre 60 y 74 años con baja escolaridad, lo cual es diferente a los resultados obtenidos en otros estudios que identifican que la menor probabilidad de vacunarse está asociada al sexo femenino, así como a un nivel bajo de estudios ${ }^{9}$ y que la probabilidad se eleva conforme avanza la edad. ${ }^{7}$ Otra diferencia significativa señala que la población urbana es más propensa a recibir la vacuna mientras que en nuestro estudio, la población es semiurbana.. El estar casado es un factor predictivo para la vacunación lo cual coincide con otros estudios revisados. ${ }^{7.9}$

En relación a la capacidad funcional, el mayor porcentaje de adultos mayores resultó ser independiente en las AVD y AIVD. No se encontraron estudios que analizaran esta variable sin embargo, el nuestro, sugiere cierta relación entre la funcionalidad y la autopercepción de buena salud, manifestada por la mayoría de quienes aceptaron la aplicación de la vacuna. En contraste, otros estudios refieren que la percepción de "mala salud" es un factor predictor para aceptar la vacunación. ${ }^{2.8}$

En nuestro estudio un porcentaje importante de la población presento comorbilidad (Diabetes Mellitus, Hipertensión Arterial y Artritis) lo que refleja una población blanco para la aplicación de la vacuna contra la influenza, como señalan diversos autores quienes refieren que la vacunación es obligada en grupos de riesgo para prevenir complicaciones, disminuir los costos y la demanda de consulta médica. ${ }^{9,10 .}$

Referente al uso de medicamentos en el momento de la encuesta, la población estudiada se encontraba consumiendo algún medicamento lo cual coincide con el estudio de Sarria ${ }^{7}$ en el que se menciona que la probabilidad de vacunarse aumentó en aquellos que en las dos últimas semanas estaban medicados.

Al analizar la asociación entre tabaquismo y probabilidad de vacunación, otros estudios refieren"." que ésta es mayor en los no fumadores, lo que coincide con nuestro estudio, donde el mayor porcentaje de individuos declaró 
no ser fumadora, y difiere con lo que al respecto reportó otra investigación?, en la cual aquellos que fumaban fueron significativamente más propensos a recibir la vacuna.

Respecto a si los adultos mayores poseen información sobre la enfermedad de la influenza y su vacuna, se encontró que un porcentaje alto de la población carece de ella; sin embargo, aceptó la aplicación de la vạcuna a diferencia de otros estudios que refieren que la ausencia de información es un factor importante para rechiazarla. ${ }^{10.12}$

El sentimiento de que la vacuna es efectiva y no causará malestar, es un predictor para la vacunación. ${ }^{13}$ Lo cual coincide con las creencias que prevalecian en la población al momento de ser estudiada: que la enfermedad es grave, la vacuna previene la influenza y que su aplicación no le ocasionaria problemas.

En México la mayoría de la población carece de una cultura adecuada sobre lo que es la influenza y de la utilización de su vacuna', no obstante, nuestro estudio refleja que esto no fué un obstáculo para la aceptación de la vacuna.

El no haber recibido la vacuna en ocasión previa, es factor predictivo para rehusarla, como lo señalan los estudios consultados. ${ }^{2}$.15 Al respecto, nuestros resultados mostraron que opuestamente, la mayor parte de la población estudiada aceptó la vacuna aún cuando nunca le habia sido aplicada, lo que denota que es posible cambiar los hábitos.

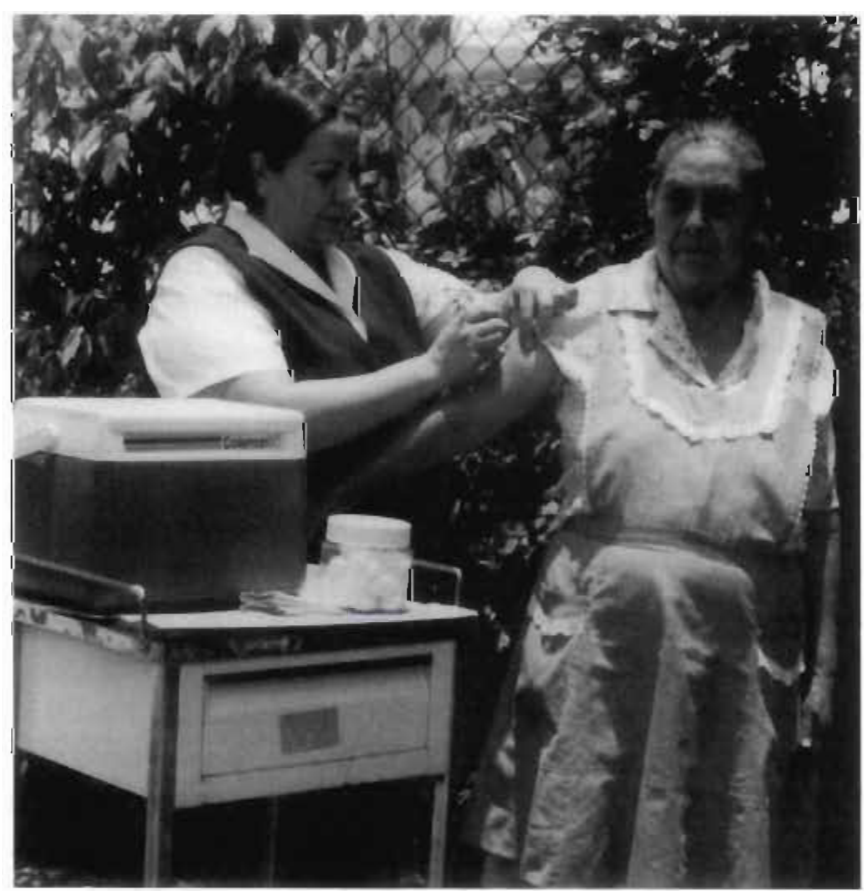

En esta investigación encontró que una minoría de adultos mayores refieren que fué el médico quien les prescribió la vacuna. Lo anterior coincide con lo que reporta un estudio, en el cual, la mayor parte de los médicos declaró no haber recibido información acerca de los beneficios de la aplicación de vacuna, por lo que no la prescribió. ${ }^{16}$ En nuestro estudio el $31.3 \%$ de la población refiere que fué la Enfermera quien le recomendó la inmunización, lo que coincide con el estudio de Puig-Barberá (1999), quien menciona que: "la orden previa de Enfermería de vacunar a los adultos mayores aumenta significativamente la vacunación antigripal en este grupo de riesgo."1'

El acceso fácil y gratuito a la vacuna fueron factores que determinaron la inmunización aún cuando, un importante porcentaje de la población estudiada refiere ser derechohabiente al IMSS, ISSSTE o servicio privado. Lo anterior sugiere, que el acceso gratuito a los servicios médicos amplía la cobertura.' En contraste con lo anterior la disponibilidad del producto se observa insuficiente ya que las dosis que circularon para la vacunación en este grupo de edad en el año 2005 fueron $2530650^{i 8}$ y según proyecciones de la CONAPO estimaron que la población nacional de los mayores de 60 años para el año 2001, eran de $7,172256.19$

\section{CONCILUSIONES}

- No existen antecedentes de estudios similares realizados en México por lo que al comparar las variables se recurrió a estudios realizados en poblaciones con caracteristicas muy diferentes (España, Canadá y Suiza). Ello explica el porqué las variables se comportaron de manera diferente y hasta contradictoria.

- Las características del adulto mayor que acepta ser vacunado contra la influenza son: ser mujer en edad entre 60 y 74 años, con pocos años de escolaridad, casada, función mental sin deterioro, no presentar depresión, auto percepción de buena salud, presentar alguna enfermedad crónico-degenerativa, buena capacidad funcional, consumir algún medicamento y no presentar el hábito de fumar.

- Los grupos de riesgo identificados para no vacunarse son: las personas mayores de 74 años, principalmente los hombres, personas con algún problema en la movilidad y no presentar alguna enfermedad crónica degenerativa, por lo que adultos mayores con las características antes descritas debieran ser objeto de futuras intervenciones a través de estrategias educativas intensas tendientes a sensibilizarlos en relación a 
la importancia y necesidad de que reciban la vacuna contra influenza y el riesgo que tienen hacia ésta enfermedad.

- El porcentaje de adultos mayores que carecía de los conocimientos acerca de la enfermedad y la vacúna también fué alto, sin embargo esto no fué determinante para rechazar la aplicación de la vacuna contra la influenza, ya que el $84.4 \%$ la aceptó, por lo que se considera que sus creencias de salud como: que la enfermedad es grave, que la vacuna previene la gripe y que su aplicación no le ocasionará problemas, influyeron en la aceptación.

- La Enfermera fué la persona que en mayor porcentaje recomendó la inmunización, lo que refleja que las intervenciones realizadas por este profesional, a través de la atención comunitaria pueden tener un impacto importante en la solución de los problemas de salud de los adultos mayores.

- Más del 68\% de los adultos mayores que no habían recibido la vacuna contra la influenza, son derechohabientes del ISSSTE ó IMSS o servicio médico particular aún no habían recibido la vacuna contra la influenza. Lo anterior refleja el dificil acceso a los servicios de salud ó la insuficiencia en la distribución de dosis de vacuna contra la influenza y de continuar con ésta tendencia se espera que la subcobertura empeore

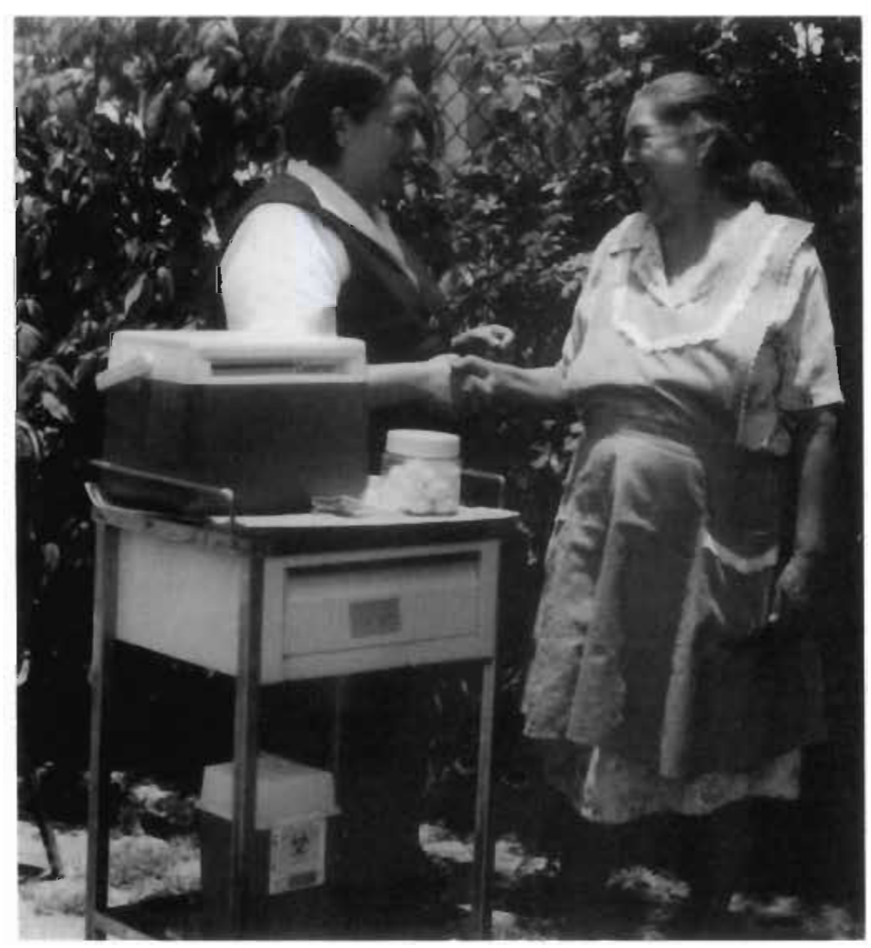

\section{REFERENCIAS BIBLIOGRAFICAS}

1. Grupo Regional de Observación de la Gripe (GROG) México Vigilancia Epidemiológica en Mexico. 2005, pp. 1 Disponible en: http://uww.ge.ocities.com/grogmx/vigilanciamex.html

2. Singleton IA y cols. Influenza, prieumococcal, and tetanus toxioid vaccination of adults-United States, 1993-7, MMWR. 2000; 49:3962.

3. Pregliasco F y cols. Influenza vaccination among the elderly in italy Bull World Health Organ 1999; 77:127-131

4. Nicholson $K G$, Kent J, Hammersley V Influenza A among communitydwelling elderly persons in Leicestershire during winter 1993-4; cigarette smoking as a risk factor and the efficacy of influenza vaccination. Epidemiol Infect 1999;123:103-108.

5. Guarda A y cols. Comparación de equipos de atención primaria de Barcelona según fórmulas de gestión. Aten. Primaria 2000; 26:6006006.

6. Egido PA y cols. Vacunación antigripal: evaluación de un programa integrado en un área básica de salud urbana. Aten. Primaria 1989; 6 : $578-582$

7. Sarriá A, Timoner J. Determinantes de la vacunación de la gripe en personas mayores de 65 años. Rev. Esp. Salud Pública 2002; $76:(1)$

8. Lawrence $\mathrm{C}$ y cols. Prevalence rate and reasons for refusals of influenza vaccine in the elderly. Swiss Medical Weekly, 2003; 133: 598-602.

9. Andrew KM y cols. Rates of influenza vaccination in older adults and factors associated with vaccine use: A secondary analysis of the Canadian Study of Health and Aging. BMC Public Health, 2004;4:36

10. Batalla 」 y cols. La cobertura de la vacunación antigripal en Cataluña. Rev. Vacunas. 2001; 2(4): 130-134.

11 . Peña-Rey I, Pérez-Farinós N, Sarria-Santamera A. Determinantes de la vacunación de la gripe en mujeres gallegas mayores de 65 años Aten Primaria 2003; 31: 4.62-463.

12. Mulet-Pons MJ y cols. Evaluación del cumplimiento de la vacunación antigripal. Aten Primaria. 1995; 16(7): 423-7.

13. Gené $\rfloor$ y cols. Do knowledge and attitudes about influenza and its immunization affect the likelifiood of obtaining immunization? Fam Pract Res J; 1992; 12(1): 61-73

14. Moreira-Rios MN. ¿Debemos promover la vacuna contra la influenza? Rev. Med. IMSS Mex. 2001; 39(1): 55-66.

15. Schwarz-Chavarria H y cols. Eficiencia de la vacunación antigripal ¿Están organizados los centros de salud para incrementar las coberturas vacunales? Aten. Primaria. 2004; 33 (09): 526-526

16. Fica CA y cols. Prescripcion de la vacuna anti influenza por médicos institucionales y estudiantes de especialidades médicas en un hospital docente, Rev. Chil. Infect 2001; 18(1): 20-27.

17. Puig-Barbera J y cols. Impacto de distintas estrategias en las tasas de vacunación antigripal en los ancianos. Aten. Primaria, 1999; 23:339-345

18. Grupo Regional de Observación de la Gripe (GROG) México, 2005 , pp. TMedidas Preventivas. Disponible en: http://unw.geocities.com/grogmx/medidas.html

19. Secretaria de Salud. Programa de Acción: Atención al Envejecimiento, 2001.pp $46 . \quad$ Disponible en: http://bvs.insp.mx/articulos/5/2/112002.pdf 12 